\title{
A New Multicast Opportunistic Routing Protocol for Wireless Mesh Networks
}

\author{
Amir Darehshoorzadeh and Llorenç Cerdà-Alabern \\ Computer Architecture Dep. \\ Univ. Politècnica de Catalunya \\ Barcelona, Spain \\ \{amir, llorenc\}@ac.upc.edu
}

\begin{abstract}
Opportunistic Routing (OR) has been proposed to improve the efficiency of unicast protocols in wireless networks. In this paper, we propose a new multicast routing protocol based on OR for wireless mesh networks, named Multicast OR Protocol (MORP). We compare our proposal with the well known ODMRP Multicast protocol.
\end{abstract}

Keywords: Opportunistic routing; Multicast; Wireless

\section{Introduction}

Opportunistic Routing (OR) has been investigated in recent years as a way to increase the performance of unicast in multi-hop wireless networks. In OR, in contrast to traditional routing, instead of preselecting a single specific node to be the next-hop forwarder, an ordered set of nodes (referred to as candidates) is selected as the next-hop potential forwarders. More specifically, when the current node transmits a packet, all the candidates that receive the packet successfully will coordinate with each other to determine which one would actually forward the packet according to some criteria, while the other nodes will simply discard the packet.

Previous research of OR mainly focused on developing various types of OR algorithms for unicast protocol and evaluating their performance $[1,11,5,9]$. Multicast OR has received relatively few attention. In [6] it used network coding to improve multicast efficiency and simplify node coordination. In [7] it is proposed an overlay multicast to adapt OR in wireless network.

In this paper we propose a new multicast routing protocol based on OR. We will refer to our proposal as Multicast Opportunistic Routing Protocol, MORP. It opportunistically employs a set of forwarders to send a packet toward all destinations. The basic ideal of MORP is to form a candidate set to reach the destinations and based on the candidates which successfully receive the packet, selects a set of candidates as the forwarders to reach all destinations. Each forwarder is responsible for sending the packet to a subset of destinations. Indeed, based on the candidates that successfully receive the packet in each transmission, MORP builds a multicast tree on the fly using OR and forwards the packet through the tree. 


\section{Multicast Opportunistic Routing Protocol}

In this section we propose a new multicast routing protocol that we call Multicast Opportunistic Routing Protocol, MORP. Assume a network with $N$ nodes and a multicast group $M$ consisting of one source $S$ and a set of destinations $\mathbf{D}=$ $\left\{D_{1}, D_{2}, \ldots, D_{n}\right\}$. Denote $C^{j, D_{i}}=\left\{c_{1}, c_{2}, \cdots c_{n}\right\}$ as the candidates set of node $j$ to reach destination $D_{i}$ ( $c_{1}$ the highest priority, and $c_{n}$ the least one), and $C^{j, \mathbf{D}}$ as the multicast candidates set of node $j$ to reach the destinations in $\mathbf{D}$.

Before a transmission starts, each node in the network must compute $C^{j, D_{i}}$ for each $D_{i} \in \mathbf{D}$, and store them in a Candidate-Table. This would be done using one of the candidates selection algorithms of the unicast opportunistic protocols that have been proposed in the literature (like ExOR [1]). Each time the source $S$ wants to transmit a packet, the following three-way-handshaking is carried out: First the source inserts its multicast candidates set $\left(C^{S, \mathbf{D}}\right)$, which is the union of all the candidates sets to reach $\mathbf{D}$ : $\bigcup_{D_{i} \in \mathbf{D}} C^{S, D_{i}}$, in the data packet and transmits it. Each candidate which successfully receives the packet sends back an acknowledgment $(\mathrm{ACK})$. After a period of time $\left(T_{A c k}\right)$ the source checks if it received ACKs from enough candidates to reach all destinations. If no enough ACKs were received, it retransmits the packet. This is done up to a maximum number of retransmissions $\left(M A X_{R e T x}\right)$. Then the source selects the candidates responsible to forward the packet (forwarding set), and chooses to which destinations each of them must care. This process is explained in section 2.1. We will refer to the set of destinations chosen for each forwarder $c_{j}$ as its Bind-Destinations, and denote it as $D_{c_{j}}^{\prime}$. Note that the Bind-Destinations for the source node is the multicast destinations set: $D_{S}{ }^{\prime}=\mathbf{D}$.

The source creates a set $\mathbf{F}$ with the IDs of the forwarding set and their BindDestinations. Then the source puts the set $\mathbf{F}$ in a control packet, that we will refer to as ForwardingPacket, and broadcast it. Each candidate $c_{j}$ that receives the ForwardingPacket and its ID is included in it, would forward the packet following the same rules as the source, except that its Bind-Destinations $\left(D_{c_{j}}^{\prime}\right)$ will be used instead of $\mathbf{D}$. This process is continued until the packet reaches the destinations.

\subsection{Candidate Coordination}

Upon transmitting a packet, the node collects the received ACKs in an AckTable. When $T_{\text {Ack }}$ expires, the forwarding set is computed as follows. If node $j$ receives an ACK from one of the candidates in set $C^{j, D_{i}}\left(D_{i} \in \mathbf{D}_{\mathbf{j}}^{\prime}\right)$, it assumes that the packet can reach $D_{i}$, and the candidate with the highest priority to reach $D_{i}$ which sent ACK is chosen as responsible to forward the packet toward $D_{i}$. Recall that the highest priority candidate is the candidate which has the least expected number of transmission to the destination. On the other hand, if $j$ does not receive any ACK from nodes in $C^{j, D_{i}}\left(D_{i} \in \mathbf{D}_{\mathbf{j}}^{\prime}\right)$, it assumes that the packet can not reach $D_{i}$ and retransmits the data packet. The node retransmits the data packet for at most $M A X_{R e T x}$ times or until receiving ACKs from enough candidates to reach all destinations. 
If $j$ finds that it is possible to reach all destinations in $\mathbf{D}_{\mathbf{j}}^{\prime}$, then it sends the ForwardingPacket announcement. In ForwardingPacket, $j$ determines which candidates would actually forward the packet, and to which destinations (BindDestinations). Note that for each destination only one candidate would be chosen to forward the packet, and the same candidate can be chosen to forward the packet to more than one destination. If the number of retransmissions of a data packet reaches $M A X_{R e T x}$ and there are not enough ACKs to reach all destinations, then the ForwardingPacket for the reachable destinations would be sent.

\section{Performance Evaluation}

\subsection{Simulation Environment}

The simulation code has been implemented within the Global Mobile Simulation (GloMoSim) library [10]. In the simulation we have modeled a network with different number of nodes $(20 \leq N \leq 100)$ placed randomly within a square with diagonal equal to $500 \mathrm{~m}$. Each simulation runs for 300 seconds of simulation time. The IEEE 802.11 Distributed Coordination Function was used as the medium access control protocol and the channel capacity was $2 \mathrm{Mbit} / \mathrm{sec}$. Each point in the plots was obtained averaging over 20 runs with different random node positions. The traffic generated by the source is Constant Bit Rate with 1 packet per second and 512 bytes of payload.

The number of multicast groups and sources is set to one in all scenarios. Destination nodes are chosen randomly. We have used different number of destinations $(2 \leq$ NumDest $\leq 10)$. Members join the multicast group at the start of the simulation and remain throughout the simulation. We have used ExOR as the candidate selection algorithm, fixing the maximum number of candidates $n c a n d=2$ (see [4] for details).

For a more realistic simulation, we have used the shadowing propagation model with parameters $\beta=2.7$ and $\sigma_{d B}=6 \mathrm{dBs}$. The delivery probabilities of the links obtained with the shadowing model were used in the candidates selection algorithm (see our previous works [2-4] for details).

To evaluate the performance of MORP, we compare it with ODMRP [8]. ODMRP is a mesh-based multicast protocol scheme and uses a forwarding group concept; only a subset of nodes forward the multicast packet via scoped flooding. It applies on-demand procedures to dynamically build routes and maintain multicast group membership. We have changed the way that ODMRP creates the routes to adapt it to the shadowing propagation model. We have evaluated both protocols as a function of number of nodes and number of destinations. The measures of interest are:

- Data Delivery Ratio: The ratio of data packets received by destinations to the number of data packets sent by the source.

- Forwarding overhead: Total number of data packets transmitted over the total number of received packets. 
- Control Packets overhead: The ratio of total number of control packets transmitted to the total number of data packets delivered.

- End-to-End delay: Average end-to-end delay of all data packets received by the destinations.

\subsection{Data Delivery Ratio}

Figure 1 shows the packet delivery ratio varying the number of nodes, but maintaining the diagonal of the area of the network equal to $D=500 \mathrm{~m}$. For each point in the figure we have added error bars at $95 \%$ confidence interval. In this figure the number of destinations have been set to $5($ NumDest $=5)$. The results are shown varying the maximum number of retransmission $\left(M A X_{R e T x}\right)$ of MORP. The legend MORP-ExOR $(n)$ in figure 1 refers to MORP with $M A X_{R e T x}=$ $n$.

We can see that using MORP with any number of $M A X_{R e T x}$ outperforms ODMRP. Even if MORP does not retransmit any data packet (MORP-ExOR(1)), it achieves about $70 \%$ packet delivery ratio, while for ODMRP the delivery ratio is about $60 \%$. This can be explained because the construction of the routes in ODMRP are subject to the random losses that may have the Join-Query packets. On the other hand, routes in MORP depends on the selection of the candidates sets, which is done taking into account the delivery probability of the links.

It is obvious that the more retransmissions are allowed in MORP, the higher will be the delivery ratio of the data packets. We can see that the differences between MORP-ExOR(1) and two other experiments (MORP-ExOR(2) and MORP-ExOR(3)) are about $17 \%$ and $23 \%$, respectively.

Figures 2 and 3 have been obtained respectively with a total number of nodes equal to $N=20$ and $N=100$, representing a low and high density network, and varying the number of destinations: NumDest $=2,3, \ldots, 10$.

Figure 3 shows that the delivery ratio of MORP-ExOR(1) for 8, 9 and 10 destinations is a bit less than ODMRP (about 2\%, 3\% and 5\%, respectively). This comes from the fact that the more destinations are, the larger are the forwarding groups in ODMRP. Therefore the packet delivery ratio in ODMRP increases by increasing the number of destinations, however, at the cost of increasing too the forwarding overhead. Nevertheless, increasing $M A X_{R e T x}$ in MORP to 2 and 3, increases the delivery ratio to $82 \%$ and $88 \%$, respectively, which outperforms the $72 \%$ obtained with ODMRP.

\subsection{Forwarding Overhead}

In this section we compare the forwarding overhead of MORP and ODMRP. Recall that we define the forwarding overhead as the total number of data packet transmissions by any node, over the total number of packets received by any destination.

Figure 4 shows the forwarding overhead varying the number of nodes in the case of 5 destinations. ODMRP periodically floods a data packet together with a Join-Query packet. I.e., it piggybacks the Join-Query information on the data 

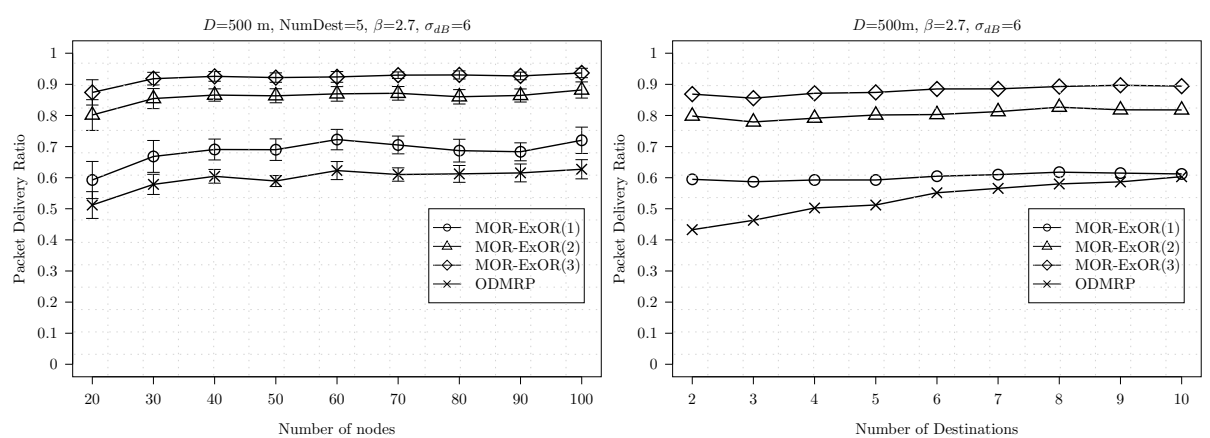

Fig. 1: Packet delivery ratio for 5 desti- Fig. 2: Packet delivery ratio with $\mathrm{N}=20$ nations nodes varying the number of destinations

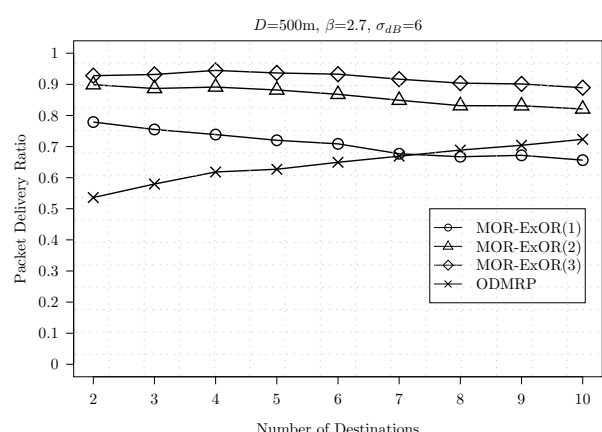

Fig. 3: Packet delivery ratio with $\mathrm{N}=100$ nodes varying the number of destinations

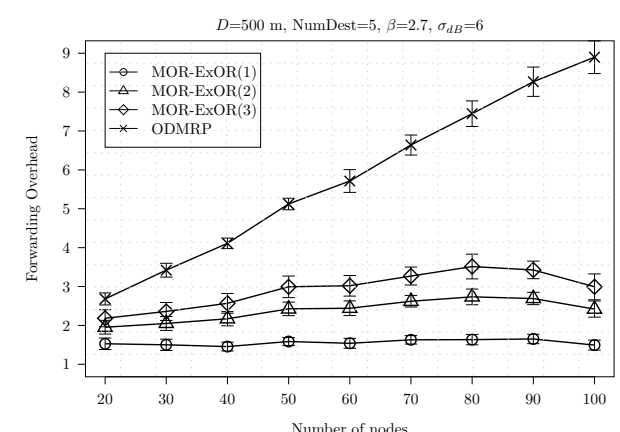

Fig. 4: Forwarding overhead for 5 destinations

packet periodically to update the membership information. For this reason the forwarding overhead of ODMRP is dominated by the flooding packets. Therefore, the higher is the node density of the network, the higher is the ODMRP forwarding overhead. On the other hand, the forwarding overhead of MORP is rather insensitive to the network density. This is because using opportunistic routing, as in MORP, only some useful nodes are selected as candidates to forward the packets, and thus, the number of forwarders is limited. Obviously, the higher is the number of retransmissions of data packets allowed in MORP, the higher will be the forwarding overhead. Nevertheless, figure 4 shows that in all cases the forwarding overhead of MORP is less than in ODMRP.

Figures 5 and 6 show more results of the forwarding overhead, varying the number of destinations for a low and high dense network. These two figures depict that in a dense network MORP is less sensitive to the number of destination. This is because having more nodes in the network, allows the candidates selection 


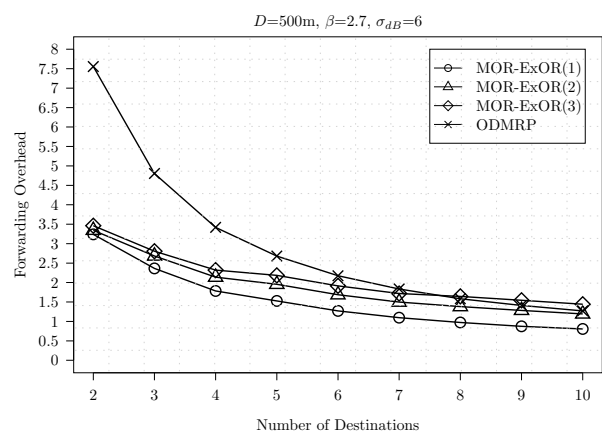

Fig. 5: Forwarding overhead varying the number of destinations. $\mathrm{N}=20$.

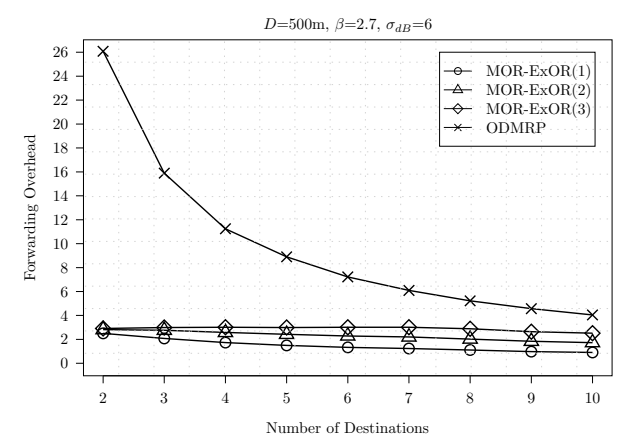

Fig. 6: Forwarding overhead varying the number of destinations. $\mathrm{N}=100$.

algorithm to better choose candidates. On the other hand, as mentioned before, the forwarding overhead in ODMRP is closely related to the network density.

Note that in figure 5 the forwarding overhead for ODMRP with 10 destinations is about 1.27 and for MORP-ExOR(2) is about 1.16. Although these two values are close to each other, figure 2 shows that the delivery ratio of ODMRP for 10 destinations is about $60 \%$ while for $\operatorname{MORP}-\operatorname{ExOR}(2)$ is about $81 \%$, so, MORP outperforms ODMRP.

On the other hand, figure 6 shows that the forwarding overhead of ODMRP with 10 destinations is about 4.04, while in MORP-ExOR(1) it is about 0.90. As we have mentioned in section 3.2 , in this case the delivery ratio in ODMRP was slightly larger than in MORP (see figure 3). We see now that this is at cost of having a forwarding overhead about 4.4 times larger than in MORP-ExOR(1).

\subsection{Control Packets Overhead}

In this section we compare the signaling overhead of MORP and ODMRP. To do so, we count as control packets for ODMRP the Join-Query, Join-Table and ACK packets, and for MORP the ForwardingPacket and ACK packets.

Figures 7 and 8 show the control overhead varying the number of destinations for low and high density networks, respectively. As the number of destinations increases, the control overhead decreases in both protocols. In MORP, the higher is the maximum number of retransmissions $\left(M A X_{R e T x}\right)$, the higher is the number of ACKs and ForwardingPackets. However, in figure 7 the control overhead of MORP in the case of $M A X_{R e T x}=2$ or 3 is only a bit higher than in ODMRP. On the other hand, its packet delivery ratio is much better than in ODMRP (see figure 2).

Additionally, figures 7 and 8 show that the control overhead of MORP with any number of retransmissions is much less sensitive to the number of destinations than in ODMRP. 


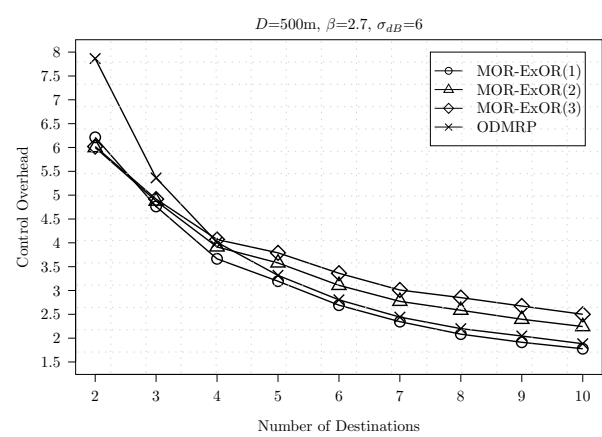

Fig. 7: Control overhead varying the number of destinations. $\mathrm{N}=20$.

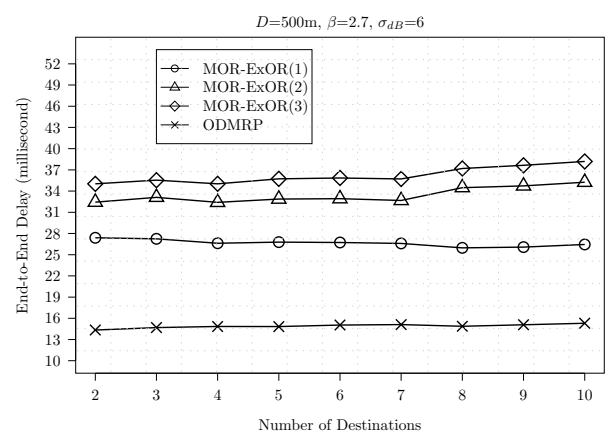

Fig. 9: End-To-End delay varying the number of destinations. $\mathrm{N}=20$.

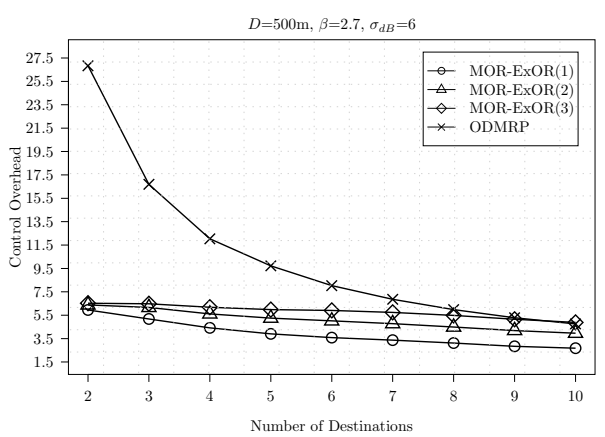

Fig. 8: Control overhead varying the number of destinations. $\mathrm{N}=100$.

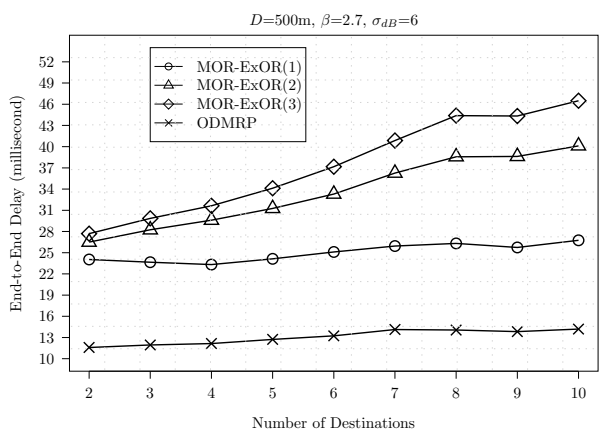

Fig. 10: End-To-End delay varying the number of destinations. $\mathrm{N}=100$.

\subsection{End-To-End Delay}

Figures 9 and 10 show the average end-to-end delay for different number of destinations with $N=20$ and $N=100$ nodes, respectively.

Recall that in MORP there is a three-way-handshaking each time a node transmits a data packet. Thus, as expected, the end-to-end delay in MORP is higher than in ODMRP. Comparing figures 9 and 10 we can see that end-to-end delay decreases in a dense network for ODMRP and MORP-ExOR(1). This is because both protocols can find better routes. For MORP-ExOR(2) and MORP$\mathrm{ExOR}(3)$, delays increase with the number of destinations. This comes from the fact that candidates set may be different to reach different destinations. Thus, more retransmissions are required until all necessary candidates receive the data packets. 


\section{Conclusion}

In this paper we propose a new multicast protocol based on opportunistic routing that we call Multicast Opportunistic Routing Protocol, MORP. MORP uses a three-way-handshaking, where the node sending the data packet chooses the forwarders and a subset of destinations to which they have to send the data packets.

We have compared our protocol with the well known mesh-based multicast routing protocol called ODMRP. In our simulations we have measured the packet delivery ratio, forwarding overhead, control overhead and end-to-end delay of both protocols.

Simulations results show that MORP outperforms ODMRP in terms of packet delivery ratio and forwarding overhead. The end-to-end delay of MORP is higher than in ODMRP, but still acceptable for real time applications. We conclude that MORP, and opportunistic routing in general, is a convenient technique to be used in multicast protocols for wireless mesh networks.

\section{Acknowledgments}

This work was supported by Spanish grants TIN2010-21378-C02-01 and 2009SGR-1167, and by the European NoE EuroNF.

\section{References}

1. S. Biswas and R. Morris. Opportunistic routing in multi-hop wireless networks. ACM SIGCOMM Computer Communication Review, 34(1):69-74, 2004.

2. L. Cerdà-Alabern, V. Pla, and A. Darehshoorzadeh. On the performance modeling of opportunistic routing. In MobiOpp '10: Second International Workshop on Mobile Opportunistic Networking, pages 15-21, New York, NY, USA, 2010. ACM.

3. L. Cerdà-Alabern, V. Pla, and A. Darehshoorzadeh. On the maximum performance in opportunistic routing. In IEEE WoWMoM 2010, Montreal, Canada, 2010.

4. A. Darehshoorzadeh and L. Cerdà-Alabern. Candidate selection algorithms in opportunistic routing. In PM2HW2N '10: 5th ACM workshop on Performance monitoring and measurement of heterogeneous wireless and wired networks, pages 48-54, New York, NY, USA, 2010. ACM.

5. H. Dubois-Ferriere, M. Grossglauser, and M. Vetterli. Least-cost opportunistic routing. In Allerton Conference on Communication, Control, and Computing, 2007.

6. D. Koutsonikolas, Y. Hu, and C.-C. Wang. Pacifier: High-throughput, reliable multicast without "crying babies" in wireless mesh networks. In INFOCOM 2009, IEEE, pages $2473-2481,2009$.

7. T. Le and Y. Liu. Opportunistic overlay multicast in wireless networks. In GLOBECOM, pages $1-5,2010$.

8. S. J. Lee, W. Su, and M. Gerla. On-demand multicast routing protocol in multihop wireless mobile networks. Mob. Netw. Appl., 7:441-453, December 2002.

9. Y. Li, W. Chen, and Z.-L. Zhang. Optimal forwarder list selection in opportunistic routing. In Mobile Adhoc and Sensor Systems. MASS '09. IEEE 6th International Conference on, pages $670-675$, oct. 2009. 
10. X. Zeng, R. Bagrodia, and M. Gerla. GloMoSim: A Library for Parallel Simulation of Large-Scale Wireless Networks. In 12th Workshop on Parallel and Distributed Simulation (PADS'98), pages 154-161. IEEE Computer Society, May 1998.

11. Z. Zhong and S. Nelakuditi. On the efficacy of opportunistic routing. In SECON '07, pages 441-450, June 2007. 